\title{
Desenvolvendo um Jogo Remoto para o ensino de Problemas de Transporte em Pesquisa Operacional
}

\author{
Thiago Schaedler Uhlmann*, Henrique Duarte Lima**, André Luiz Luppi***, Mateus Isaac Di Domenico****, \\ Luciano Antonio Mendes ***** \\ *Pontificia Universidade Católica do Paraná; e-mail: tsu@tsu-it.com \\ **Pontificia Universidade Católica do Paraná; e-mail: duarte.lima@pucpr.br \\ *** Pontificia Universidade Católica do Paraná; e-mail: luiz.luppi@pupr.br \\ **** Pontifícia Universidade Católica do Paraná; e-mail: mateus-isaac@hotmail.com \\ ***** Pontificia Universidade Católica do Paraná; e-mail: l.mendes@pucpr.br
}

\begin{abstract}
Distance Learning is an increasingly present reality in engineering courses, and automation plays a key role in enabling remote experiments, or weblabs. In these experiments, the student operates, remotely, experiments, visualizing their performance remotely through cameras and sensors. The main objective of this paper is to describe the development process of a weblab formatted as an educational game, called Steamlog, which simulates situations of rail freight transport whose solution to the issues generated is given through Linear Programming algorithms. The game is intended for graduate students in Production Engineering. The article begins with the theoretical basis necessary for the design and construction of the game. Afterwards, the game development is described contemplating the control system, based on ISA - iLab Shared Architecture, and the automation of the components of the game train and cargo stations, operated through MQTT protocols with the use of Internet of Things. Finally, the aforementioned elements and systems are tested individually for viability and functionality. The article concludes by pointing out that Steamlog is still under development, and that additional testing is required until a fully functional prototype is complete and ready for use.
\end{abstract}

Resumo: O Ensino à Distância é uma realidade em consolidação nos cursos de engenharia. Experimentos remotos, ou weblabs, têm se mostrado importantes recursos de apoio para incrementar o valor pedagógico da modalidade. Nos experimentos operados à distância, o aluno opera dispositivos ou plantas de laboratório com fins didáticos, visualizando-os remotamente por meio de imagens de câmeras, monitorando sensores e enviando comandos a atuadores. Com os mesmos recursos de automação, podese realizar também jogos remotos, a combinação entre weblabs e jogos sérios educacionais. Neste trabalho, descreve-se o processo de desenvolvimento de um experimento no formato de jogo educacional, denominado Steamlog, com a finalidade de realizar em pequena escala operações de logística do modal de transporte ferroviário de cargas. O jogo remoto é simultaneamente operado por duas equipes, permitindo aplicar em contexto prático soluções para problemas clássicos da engenharia de produção como o de fluxo de custo mínimo e o do caixeiro viajante. O artigo trata da fundamentação teórica utilizada no projeto, da integração dos componentes do jogo - trens, cargas e estações de transbordo, operados desde um client na internet e localmente conectados por meio de dispositivos microprocessados programáveis. Um servidor local encarregado da mediação de comandos do usuário e envio de dados à interface remota segue a arquitetura ELSA, uma extensão da MIT-ISA usada no gerenciamento de utilização de experimentos remotos. As soluções combinadas para implementar as funcionalidades do jogo resultaram em uma integração satisfatória para atender aos requisitos do projeto.

Keywords: Remote Games, Game-Based Learning, Transport and Distribution Logistics, Linear Programming, iLab Shared Architecture.

Palavras-chaves: Jogos Remotos, Aprendizagem Baseada em Jogos, Logística de Transporte e Distribuição, iLab Shared Architecture.

\section{INTRODUÇÃO}

O Ensino à Distância (EAD) constitui-se em uma realidade cada vez mais presente no Ensino Superior no Brasil e em vários países. Especificamente, no ensino de Engenharia, onde se faz necessário a demonstração de conceitos e teorias por meio de laboratórios, a automação e 0 uso da comunicação por rede (ex.: Internet) se fazem presentes e necessárias para que esta prática laboratorial seja possível à distância, e a interação aluno e professor seja minimamente prejudicada em relação à prática presencial.

O presente artigo possui como objetivo principal descrever o processo de desenvolvimento de um experimento remoto 
automatizado, no formato de um jogo (jogo remoto), com o propósito de replicar em um cenário situações de transporte ferroviário de cargas, sendo tais situações solucionáveis por meio de algoritmos de programação linear e otimização. O jogo remoto Steamlog, artefato que busca atender à proposta descrita anteriormente, encontra-se na fase de integração de componentes.

Neste jogo, uma ou duas equipes comandam operações de movimentação a transporte de cargas de trens em uma malha ferroviária em escala. A utilização à distância, por alunos e professores, é organizada por meio de um sistema de gestão de acesso.

O jogo teve seus componentes definidos e desenvolvidos com vistas aos aspectos teóricos relacionados a dois conceitos pedagógicos de crescente relevância no universo educacional: a Experimentação Remota e a Aprendizagem Baseada em Jogos, ambos concordantes com os conceitos de Metodologias Ativas de Aprendizagem.

$\mathrm{O}$ artigo se encontra estruturado em três tópicos principais. Primeiramente, descrevem-se os aspectos teóricos necessários para a conceituação, o projeto e a construção do jogo remoto Steamlog. A seguir, descreve-se o processo de desenvolvimento deste jogo remoto contemplando o gerenciamento de acessos via Internet, além do funcionamento do sistema como um todo e de seus componentes. Finalmente, testes de funcionamento do jogo são descritos de forma a demonstrar, de forma parcial, a viabilidade deste jogo remoto como sistema automatizado de aprendizagem, assim como aplicações futuras desse jogo no ambiente acadêmico.

\section{ASPECTOS TEÓRICOS}

Para o desenvolvimento do jogo remoto Steamlog foi realizada pesquisa exploratória em relação aos principais conceitos educacionais que deveriam direcionar a definição de funcionalidades e, consequentemente, a seleção de componentes para consecução. Estes conceitos são o de Experimentação Remota, Aprendizagem Baseada em Jogos e Programação Linear (em especial, aplicada aos problemas de transporte).

A Experimentação Remota, como o nome sugere, consiste na prática da experimentação laboratorial à distância, por meio de experimentos remotos (também conhecidos como laboratórios remotos ou weblabs), acessados e controlados à distância por professores e estudantes. Conforme Nickerson et al. (2007), um experimento remoto deve ser avaliado sob diferentes aspectos, sendo alguns estes: o objetivo para o qual este se destina, sua interface (tanto para o usuário como para os administradores), o formato e tecnologias utilizadas neste laboratório, e aspectos relacionados ao usuário como indivíduo.

No presente caso, a experimentação remota foi combinada com outro paradigma educacional, o da Aprendizagem Baseada em Jogos (Hamari et al. 2016). Tal combinação foi justificada tendo em vista estudo realizado por Bodnar et al. (2016) que, por meio de uma revisão sistemática, constatou o crescente uso de jogos digitais, jogos não-digitais e outras práticas lúdicas em diferentes campos da Engenharia, tais como as Engenharias de Produção, Civil, Mecânica, de Computação, dentre outras. Há relato do uso de elementos lúdicos em laboratório remoto em um experimentos de interação com robôs seguidores de linha (Iturrate et al. 2013)

Operações de transporte ferroviário de cargas definem problemas que podem ser resolvidos por meio de algoritmos de Programação Linear. Em particular, o problema do Fluxo de Custo Mínimo é abordado em disciplinas ligadas à Logística em cursos de Engenharia de Produção, na forma de um problema de otimização linear de transporte onde buscase a minimização dos custos considerando-se nós demandantes e ofertantes (Hillier e Lieberman, 2013). Este modelo se encontra resumido abaixo.

$$
\begin{gathered}
\text { Minimizar } \boldsymbol{Z}=\sum_{i=1}^{n} \sum_{j=1}^{n} c_{i j} x_{i j} \\
x_{i j}=\text { fluxo por meio do trajeto } i \rightarrow j \\
c_{i j}=\text { custo por fluxo por meio do trajeto } i \rightarrow j \\
\quad \text { sujeito a } \\
\sum_{j=1}^{n} x_{i j}-\sum_{j=1}^{n} x_{j i}=\boldsymbol{b}_{i} \text { (para cada nó } i \text { ) } \\
\text { sendo que } \\
b_{i}>0 \text { se o nó } i \text { for um nó de suprimento } \\
b_{i}<0 \text { se o nó } i \text { for um nó de demanda } \\
b_{i}=0 \text { se o nó } i \text { for um nó de transbordo }
\end{gathered}
$$

As situações-problema relacionadas com logística de transporte podem ser incrementadas em complexidade com a adição de operações de movimentação local de estoques.

A adição de tais desafios vai ao encontro de elementos essenciais para que um jogo sério, no contexto da Educação à Distância, atinja o seu objetivo. $\mathrm{Fu}, \mathrm{Su}$ e $\mathrm{Yu}$ (2009) enumeram alguns destes elementos, sendo estes: a concentração (o jogador se manter concentrado no jogo), a clareza de metas (o jogador saber o que deverá realizar), a retroalimentação ou feedback (ele ter o retorno das suas ações por parte do sistema), os desafios apresentados (devem motivar o jogador), a autonomia (sentimento de controle por parte do jogador) e a imersão (envolvimento do jogador pelos cenários, narrativa e demais elementos do jogo).

\section{METODOLOGIA}

O presente projeto pode ser abordado como objeto das Ciências do Design, ou Design Sciences (Van Aken, 2005), envolvendo fases de concepção, desenvolvimento e operacionalização de um produto ou sistema ante requisitos específicos. De modo geral, dentre as possíveis formas de se solucionar o problema de projeto (Dresch, Lacerda e Miguel, 2015) busca-se aquela que melhor se equilibra entre os recursos disponíveis e as restrições presentes. O método adotado no desenvolvimento do Steamlog foi delineado em quatro etapas, como segue. 
A primeira etapa consiste no projeto informacional. Foram definidos os requisitos do jogo, do usuário e técnicos, alcançando os elementos lúdicos (mecânicas de jogo, narrativa, fluxo de jogo, regras, etc.), e aspectos estruturais (necessidades de componentes, tecnologias a serem utilizadas, dentre outros).

A segunda etapa contempla o projeto de conceitos, dimensionamentos, construção e integração de subsistemas e a sua integração, correspondentes aos elementos selecionados e criados de hardware, módulos de software e componentes mecânicos especiais. Desta forma, foram criados estações, trens e a malha ferroviária. Uma maquete cenográfica foi adicionada para maior imersão do jogador no contexto. As funcionalidades para operação remota simultânea por duas equipes foram criadas em acordo com a arquitetura ISA/ELSA, da forma descrita adiante. Nesta fase do desenvolvimento, alguns requisitos técnicos foram ajustados quando em benefício dos propósitos do jogo.

A terceira etapa, em concomitância com a segunda, contempla a realização de testes de integração progressiva dos componentes e com a interface remota de operação.

Por ora da redação deste artigo, não tem-se ainda dados da quarta etapa, correspondentente à realização de testes com usuários, previstos para o futuro próximo.

\section{JOGO STEAMLOG SOB A ARQUITETURA ISA/ELSA}

O labserver é acessado por meio de um sistema de gerenciamento e controle de acessos, o ISA - iLab Shared Architecture (Harward et al. 2008). Por meio deste sistema, as equipes de jogo efetuam o agendamento de horários, dentre os definidos pelo administrador (professor da disciplina) para acesso ao jogo remoto. $\mathrm{O}$ acesso à interface somente é disponibilizado por ora do início do jogo, por meio de um redirecionamento a um servidor de internet na nuvem.

Para os labservers utiliza-se a arquitetura ELSA-SP (Uhlmann et al. 2019), rodando um microcomputador Raspberry $\mathrm{Pi}$ 3, que atua como intermediário de dados e hospeda o código de software específico para o funcionamento de um determinado experimento (se desejado, esta atribuição pode ser transferida a outro periférico com capacidade computacional, a exemplo de uma placa Arduino ou similar). Os comandos enviados desde a interface são processados e encaminhados aos componentes do jogo remoto por meio dos códigos na RPi-3.

Os comandos aos dispositivos microprocessados localizados em um vazio sob o nível físico da malha na maquete ferroviária são transmitidos por meio de interfaces de comunicação serial. Os trens de cada composição ferroviária são dotados de módulos NodeMCU, que se comunicam por meio de uma rede wifi local exclusiva (wireless). A figura 1 ilustra a arquitetura ELSA-SP adaptada ao jogo remoto Steamlog.

\section{STEAMLOG: DETALHES DO PROCESSO DE DESENVOLVIMENTO}

O jogo remoto Steamlog está sendo desenvolvido e instalado em um laboratório de automação e manufatura nas dependências de uma universidade brasileira.

$\mathrm{Na}$ malha ferroviária do jogo, as composições transportam módulos de carga (pastilhas identificadas por etiquetas de RFID e em diferentes cores, dotadas na parte superior de um inserto ferromagnético para captura por eletroimã) entre estações (nós) ofertantes e demandantes automatizadas, que efetuam operações de transbordo de cargas entre os trens e as estações, bem como movimentações locais de estoque nas mesmas.

Cada estação possui cinco compartimentos com capacidade de empilhamento para três módulos de carga. A figura 2 ilustra a estação de carga e descarga.

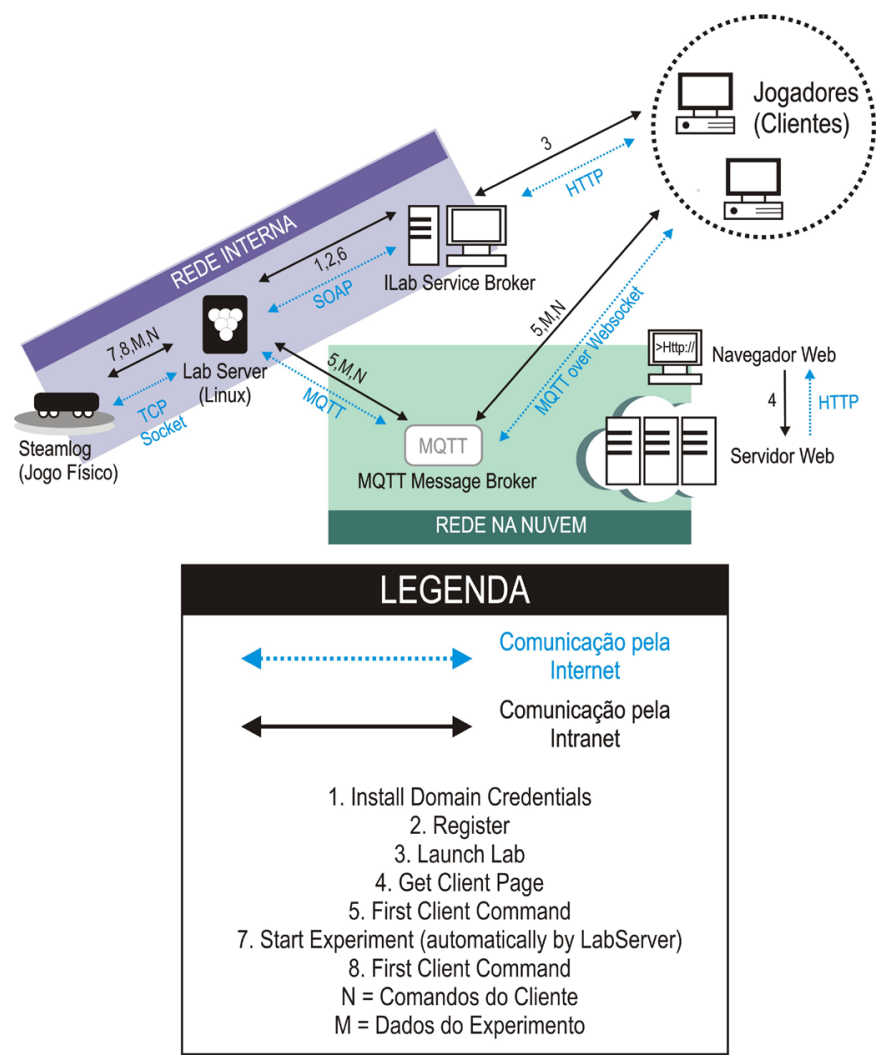

Fig. 1 Arquitetura ELSA-SP adaptada para o jogo remoto Steamlog 


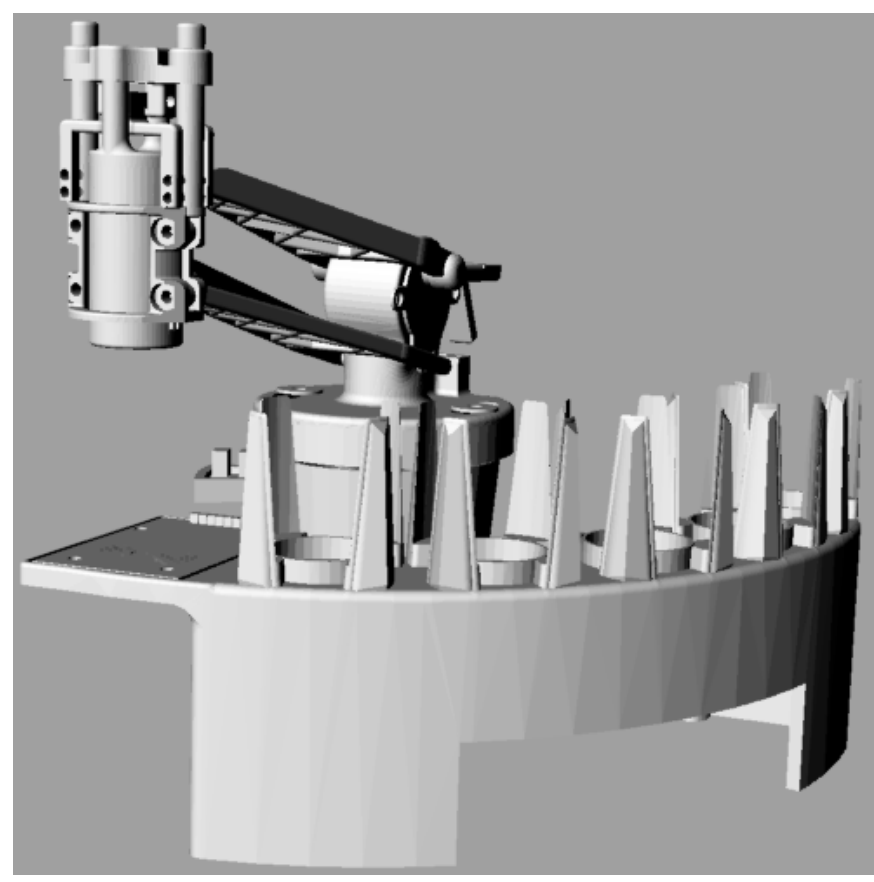

Fig. 2 Estação de Carga e Descarga: desenho esquemático

O traçado da malha ferroviária do jogo remoto foi elaborado tendo-se em vista a possibilidade da exploração do problema do Fluxo de Custo Mínimo, neste caso, o transporte de cargas por modal ferroviário para pontos demandantes a partir de pontos ofertantes, por meio de um trajeto com extensão e custo mínimos, sendo tal exploração em diferentes níveis de dificuldade. A malha em escala é composta por trilhos e interseções controladas por meio de circuitos eletrônicos de controle. O controle da movimentação dos trens é feito com entroncamentos acionáveis e sensores sob os trilhos com capacidade de identificação de trens e vagões. Unidades microprocessadas Arduino, programadas com protocolos específicos de comunicação por interface serial, foram usadas para simplificar a integração da malha e das seis estações com o LabServer na Raspberry Pi. A figura 3 ilustra a malha ferroviária, a localização das estações e das placas de controle. A circulação é permitida somente no sentido horário.

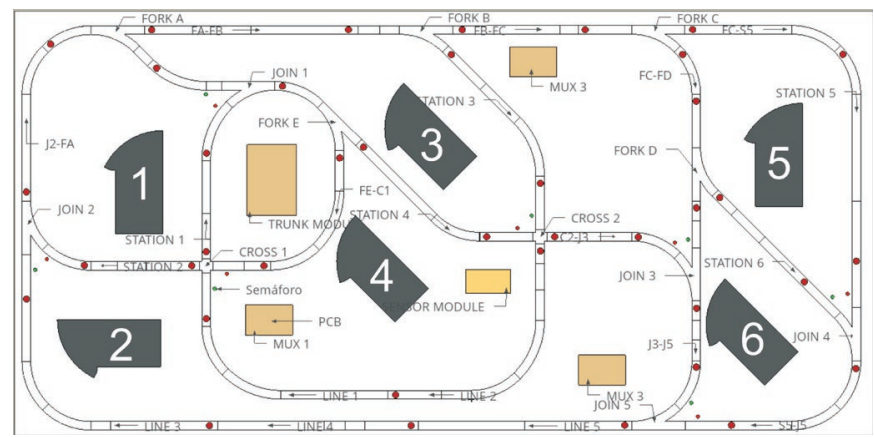

Fig. 3 Malha ferroviária: desenho esquemático (estações em cinza-escuro e placas de controle em bege)

O mapeamento da distribuição de estações, com relação às conexões diretas entre nós resulta no diagrama da Figura 4.

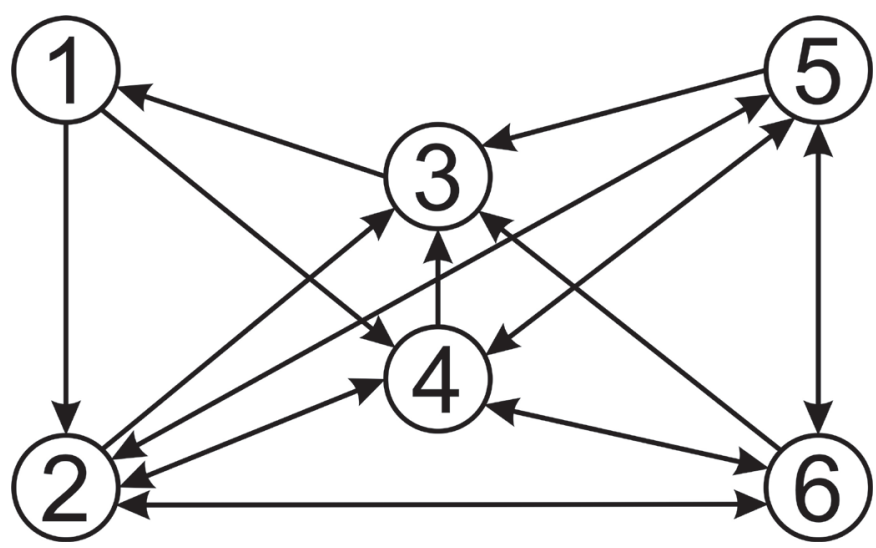

Fig. 4 Conexões diretas entre nós

A malha assim configurada, quando percorrida simultaneamente por dois trens, produz eventualmente situações de espera em confluências, estações e trilhos alocadas antes pela contraparte, devido aos automatismos presentes para impedir colisões. Adicionalmente, pode ser habilitado um segundo componente para incrementar o grau de imprevisibilidade da disponibilidade dos recursos da malha, na forma de bloqueios temporários de pontos da malha. Os bloqueios são informados tempestivamente aos usuários com pretextos do jogo por um sistema de broadcast de boletins, um canal disponível também às equipes para negociar ou combinar opções entre si. Estes elementos replicam uma dinâmica naturalmente existente no mundo real e estimulam as equipes a realizarem reprogramações oprtunas de movimentos, elevando a jogabilidade.

A estrutura integrada pode comportar variantes de conjuntos de regras e modos de jogo. A operação pode ser definida como competitiva ou colaborativa - nesta última, podem ser evidenciadas as vantagens de um planejamento conjunto.

As regras de jogo acerca do problema do Fluxo de Custo Mínimo poderão ser definidas considerando-se para a formatos diferentes de roteirização. As tarefas/objetivos podem ter complexidade variável, com múltiplos pick-up's e drop off's de cargas

- Origem e destino coincidentes: neste caso, o trem deverá, incialmente distribuir e depois recolher cargas de volta ao nó de partida. Em um exemplo, esta narrativa se encaixa no caso de produtos que devem ser distribuídos aos clientes para uso consignado por um período e então recuperados pela empresa proprietária;

- Um ponto de origem e um ponto de destino: neste caso, o desafio é descobrir o caminho mais eficaz antes de cada início de viagem, visto que o cenário pode se alterar entre as corridas. Este caso pode corresponder a um serviço de entrega periódica de matéria-prima a uma fábrica; 
- Pontos de origem e destino múltiplos: neste caso, estabelecem-se combinações entre diferentes pontos ofertantes e demandantes. Uma analogia possível seria um conjunto de fornecedores sistemistas interdependentes preparando subconjuntos de um produto final.

Regras de custeio operacional podem ser atribuídas aos movimentos do trem (distância percorrida, velocidade de tráfego, quantidade transportada, consumo de combustível) e às estações (tempo de parada do trem, transbordo de cargas, movimentos do estoque na estação).

Assim, o Steamlog pode ser aplicado como uma solução possível para a simulação de operações de transporte de cargas com o uso de modal ferroviário. Por meio deste jogo, alunos de cursos de Engenharia de Produção podem simular algoritmos de Pesquisa Operacional aplicados ao transporte, visualizando de forma prática os resultados dos cálculos efetuados.

Primeiramente, o professor disponibiliza aos alunos o ambiente de jogo, de onde será possível a visualização dos nós ofertantes e demandantes, bem como a quantidade de cargas ofertadas e demandadas por tipo, para cada nó.

Após, cada aluno (ou equipe), fora do ambiente de jogo (ex.: com um papel e caneta, ou software), efetua os cálculos relativos ao problema do Fluxo de Custo Mínimo, para descobrir a melhor rota de transporte.

A seguir, os alunos (ou equipes) comandam seus respectivos trens para coletar e transportar as cargas em uma sequência de coletas e entregas conforme o resultado de seus cálculos. Ganha a equipe que efetuar essa operação no menor tempo (o que seria o menor custo de transporte neste caso).

Tendo em vista as definições supracitadas, os componentes do jogo que já foram concluídos estão sendo progressivamente testados.

\section{STEAMLOG: TESTES DE FUNCIONAMENTO}

Testes bem sucedidos de funcionamento individual de subsistemas e sua integração já foram realizados para: manobras da malha ferroviária (acionamentos das confluências e bifurcações); monitoramento de sensores infra-vermelho analógicos de passagem e identificação dos trens localizados sob os trilhos, com partidas e paradas em locais programados mediante comandos passados via rede wi-fi; movimentos do guindaste de transbordo da estação piloto, acionamento do seu eletroímã e reconhecimento de cargas por meio de identificação RFID; elementos de segurança intrínseca, como o disjuntor de alimentação dos motores do guindaste em caso de impacto mecânico e fusíveis contra curto-circuito nos trilhos; habilitação dos labservers ELSA-SP para cada experimento remoto no sistema ISA, cada qual intermediando a operação do respectivo trem (um por equipe), bem como um terceiro para o professor monitorar o andamento da operação e eventualmente interferir; interfaces funcionais de programação de movimentos pelas equipes; integração com os sistemas de gerenciamento ISA e ELSA-SP (sendo estes usados para acesso à interface de controle e passagem de comandos ao trem e malha ferroviária).

Os web sockets entre os labservers e a interface cliente permitem transferir dados de parte a parte através de um serviço MQTT - Message Queieing Telemetry Transport, um protocolo de comunicações destinado a dispositivos móveis, como os dispositivos IoT - Internet of Things (Internet das Coisas), em redes de alta latência, localizado na nuvem, com desempenho suficiente para a aplicação. A figura 5 apresenta em vista parcial compontes do jogo remoto em processo de construção.

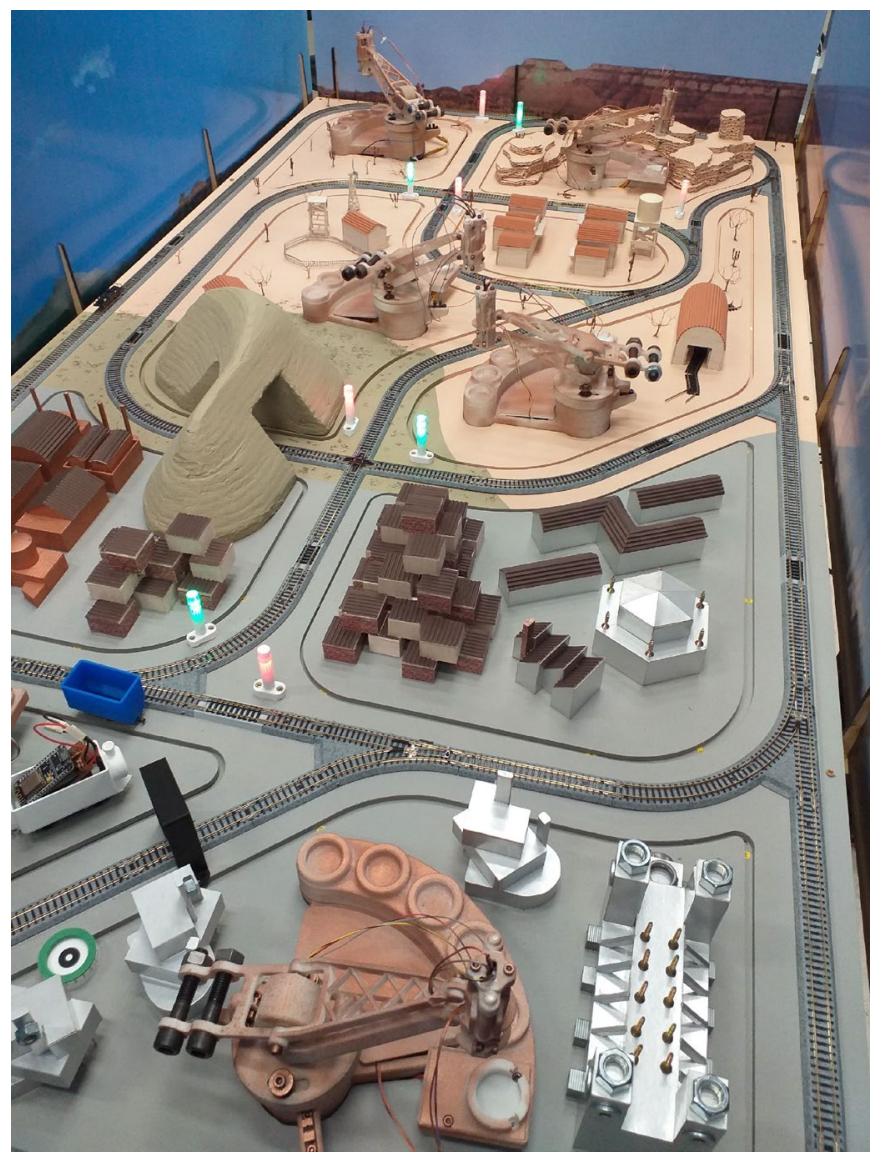

Fig. 5. Maquete do jogo Steamlog (em processo de construção)

\section{CONSIDERAÇÕES FINAIS}

O artigo descreveu o processo de concepção, desenvolvimento e implantação de um jogo remoto para a simulação de operações de transporte ferroviário de cargas, sendo tais operações efetuadas com base no problema de Fluxo de Custo Mínimo.

Conforme previsto na metodologia, três das quatro etapas do método de Ciência do Design foram realizadas. Foram definidos os requisitos técnicos do jogo (no caso, o conceito do jogo, a sequência de uso do jogo pelo professor, as arquiteturas a serem usadas (ISA e ELSA-SP). Após, o jogo foi construído tendo em vista o cumprimento dos requisitos 
definidos anteriormente. os quais foram tangibilizados na construção deste, e testes preliminares foram realizados com relação ao funcionamento dos componentes.

Os testes realizados com relação aos sistemas de controle, e os componentes projetados para o jogo, demonstraram sucesso até o momento. Porém, a integração do jogo remoto, contemplando sistemas e componentes, ainda se encontra em estágio parcial. Uma maquete cenográfica do jogo foi construída, e alguns componentes, dentre os quais a estação de carga e descarga, foram produzidos.

Assim, além dos testes relativos ao funcionamento dos componentes de forma individual, descritos neste artigo, são necessários testes adicionais para o funcionamento do sistema como um todo, e da viabilidade deste como jogo, contemplando aspectos como o cumprimento das finalidades pedagógicas para o qual foi destinado e a capacidade de prover diversão aos jogadores.

O jogo ainda se encontra em fase de desenvolvimento, com conclusão prevista para o final do segundo semestre de 2019, quando será testado como ferramenta de ensino e aprendizagem na disciplina de Pesquisa Operacional do curso de Engenharia de Produção de uma universidade.

\section{AGRADECIMENTOS}

Esta pesquisa, bem como seus resultados, recebeu o apoio das seguintes agências de fomento: Fundação Araucária (CP 09/16), CNPq (315513/2018-7) e FINEP.

\section{REFERÊNCIAS}

Bodnar, C. et al. (2016) Engineers at play: games as teaching tools for undergraduate engineering students. Journal of Engineering Education 105, 147-200.

Dresch, A., Lacerda, D. P. e Miguel, P. A. C. (2015) A Distinctive Analysis of Case Study, Action Research and Design Science Research. Review of Business Management, v. 17, No. 56, p. 1116-1133.

Fu, F., Su, R., e Yu, S. (2009) EGameFlow: A scale to measure learners' enjoyment of e-learning games. Computers \& Education 52, p. 101-112.

Hamari, J.; Shernoff, D. J.; Rowe, E.; Coller, B.; AsbellClarke, J. e Edwards, T. (2016) Challenging games help students learn: an empirical study on engagement, flow and immersion in game-based learning. Computers in Human Behavior 54, p. 170-179.

Harward, V. J. et al. (2008) The iLab Shared Architecture: A Web Services Infrastructure to Build Communities of Internet Accessible Laboratories. Proceedings of the IEEE, v. 17, i. 6.

Hillier, F. S.; Lieberman, G. J. (2013) Introdução à pesquisa operacional. Porto Alegre: AMGH.

Iturrate, I. et al. (2013) A Mobile Robot Platform for Open Learning based on Serious Games and Remote Laboratories. 1st International Conference of the Portuguese Society for Engineering Education (CISPEE).

Nickerson, J. V.; Corter, J. E.; Esche, S. K.; Chassapis, C. (2007) A model for evaluating the effectiveness of remote engineering laboratories and simulations in education. Computers \& Education 49, p. 708-725.

Uhlmann, T.S.; Mendes, L.A. (2018) Desenvolvimento de um framework para Criação de Experimentos e Jogos Remotos Cooperativos, e de um Jogo Remoto para a Simulação de Processos em Logística de Transporte e Distribuição. Projeto submetido à $<$ REVISAO CEGA $>$ como parte do Edital 07/2017 (Jogos e Simulações) para obtenção de recursos financeiros da FINEP.

Uhlmann, T.S.; Lima, H.D.; Luppi, A.L.; Mendes, L.A.; (2019) ELSA-SP: Through-the-cloud subscribe-publish scheme for interactive remote experimentation under iLab Shared Architecture and its application to an educational PID control plant. Exp'at'19 - 5th Experiment@International Conference 2019.Artigo em fase de publicação.

Van Aken, J. E. (2005) Management research as a design science: articulating the research products of mode 2 knowledge production in management. British Journal of Management, v. 16, n. 1., p. 19-36. 